\title{
A FAST ELLIPSE/CIRCLE DETECTOR USING GEOMETRIC SYMMETRY
}

\author{
CHUN-TA HO and LING-HWEI CHEN \\ Department of Computer and Information Science, National Chiao Tung University, Hsinchu, Taiwan \\ 30050, Republic of China
}

(Received 24 November 1993; in revised form 3 June 1994; received for publication 28 June 1994)

\begin{abstract}
Through the use of a global geometric symmetry, a fast ellipse/circle detector is proposed in this paper. Based on the geometric symmetry, the proposed method first locates candidates of ellipse and circle centers. In the meantime, according to these candidate centers, all feature points in an input image are grouped into several subimages. Then, for each subimage, by using geometric symmetry again, all ellipses and circles are obtained. The method significantly reduces the time required to evaluate all possible parameters without using edge direction information. Experimental results are given to show the correctness and effectiveness of the proposed method.
\end{abstract}

Ellipse detection Circle detection Geometric symmetry

\section{INTRODUCTION}

The detection of circles and ellipses from a digital image is important in industrial applications. The Hough transform ${ }^{(1)}$ has long been recognized as a robust technique for detecting line features, but applying it to detect circles and ellipses requires a threedimensional (3D) array and a 5D one, respectively, for the accumulator. Moreover, a great deal of computing time is needed to transform a feature point in an input image to many points in the parameter space. Several modified versions of Hough transform have been proposed. Tsuji and Matsumoto ${ }^{(2)}$ first introduced the decomposition concept by the use of parallel tangents. Ballard ${ }^{(3)}$ presented a method which simplifies the parameterization by deriving the ellipse center from the coordinates and tangent of an edge pixel. Illingworth et al ${ }^{(4,5)}$ proposed a better center finding process and used adaptive Hough transform to estimate three other parameters. Yip et al.$^{(6)}$ presented an approach which utilizes parallel edge points to deduce parameters. The methods mentioned above utilize gradient information to reduce the dimension of the parameter space, and the problem is broken down into multiple stages. But, estimates of the parameters of ellipses or circles based on local geometric properties often suffer from poor consistency and locating accuracy because of noise and quantization error. To avoid these disadvantages, in this paper, we will present a new method that uses the global geometric symmetry of ellipses and circles to reduce the dimension of the parameter space. Moreover, through a point-topoint transform, the proposed method can greatly reduce the computing time required for candidate parameter evaluations without losing accuracy.

In the proposed method, first a global geometric symmetry is used to locate all possible symmetric centers of ellipses and circles in an image, and then all feature points are classified into several subimages according to these center points. Ellipses and circles with different symmetric centers will lie in different subimages. Then the geometric symmetry is applied again in each subimage to find all possible sets of three parameters (the length of the major axis, the length of the minor axis and orientation) for ellipses or one parameter (radius) for circles. Finally, the accumulative concept of the Hough transform is used to extract all ellipses and circles in the input image.

The remainder of this paper is organized as follows. The proposed method is described in detail in Section 2 , and experimental results are presented in Section 3. Section 4 discusses our results and concludes the paper.

\section{PROPOSED METHOD}

The proposed method consists of two phases: symmetric center location and parameter estimation. In Phase 1, we will locate the candidates of ellipse and circle centers and classify the feature points in an input image into different subimages according to these candidate centers. In Phase 2, for each subimage, based on the geometric symmetry of ellipses and circles all ellipses and circles will be extracted. Before describing these two phases in detail, we will first introduce some theorems which will be used in the proposed method.

\subsection{Properties for ellipses and circles}

Theorem 1. Let $\mathrm{E}$ be an ellipse or circle that is scanned from left to right and top to bottom (rightward). Assume that each horizontal scan line $\mathrm{HS}_{i}$ intersects $\mathrm{E}$ at $\mathrm{XL}_{i}$ and ${X R_{i}}_{i}$ Let $X_{i}$ be the midpoint of $X_{i}$ and $X R_{i}$. Then each $\mathrm{XM}_{i}$ lies on the same straight line $l_{v}$, which will be 

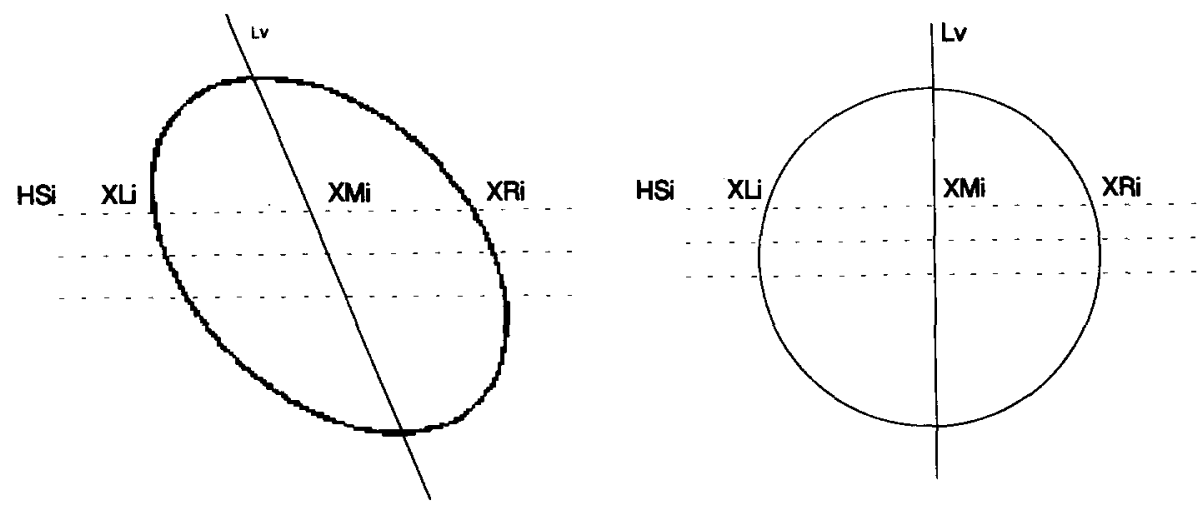

(a)
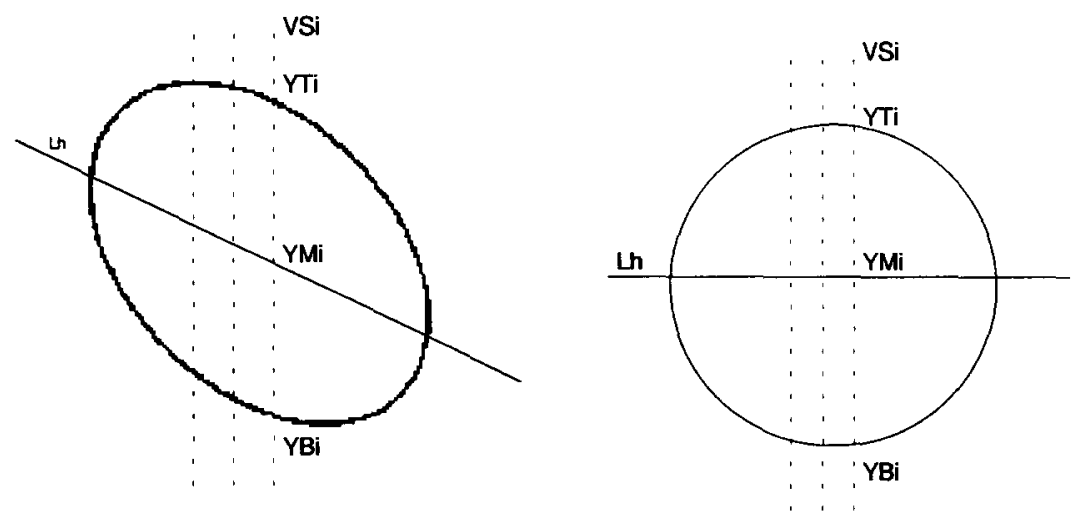

(b)

Fig. 1. The two symmetric axes generated by horizontal and vertical scanning transform: (a) the middle point $\mathrm{XM}_{i}$ of $\mathrm{XL}_{i}$ and $\mathrm{XR}_{i}$ lies on the same straight line $L_{v}$ (b) The middle point $Y M_{i}$ of $Y T_{i}$ and $Y B_{i}$ lies on the same straight line $L_{h}$.

referred to below as the symmetric vertical axis [see Fig. 1(a)].

This theorem has been proven by Yin and Chen. ${ }^{(7)}$ Note that in the sequel, $\mathbf{X R}_{i}\left(\mathrm{XL}_{i}\right)$ will be referred to as the symmetric point of $\mathrm{XL}_{i}\left(\mathrm{XR}_{i}\right)$ relative to $l_{v}$.

Theorem 2. Let $\mathrm{E}$ be an ellipse or circle that is scanned from top to bottom and left to right (downward). Assume that each vertical scan line $\mathrm{VS}_{i}$ intersects $\mathrm{E}$ at $\mathrm{YT}_{i}$ and $\mathrm{YB}_{i}$. Let $\mathrm{YM}_{i}$ be the midpoint of $\mathrm{YT}_{i}$ and $\mathrm{YB}_{i}$. Then each $\mathrm{YM}_{i}$ lies on the same straight line $l_{\mathrm{h}}$, which will be referred to below as the symmetric horizontal axis [see Fig. 1(b)].

The proof of Theorem 2 is similar to that of Theorem 1. In the sequel, $\mathrm{YT}_{i}\left(\mathrm{YB}_{i}\right)$ will be referred to as the symmetric point of $\mathrm{YB}_{i}\left(\mathrm{YT}_{i}\right)$ relative to $l_{\mathrm{h}}$.

Theorem 3. Let $\mathrm{E}$ be an ellipse or a circle and let $l_{\mathrm{v}}, l_{\mathrm{h}}$ be its two symmetric axes generated by rightward and downward scanning. Then the cross-point of $l_{\mathrm{v}}$ and $l_{\mathrm{h}}$ is the center of $E$.

Proof. Without loss of generality, suppose that the center point of $E$ is $(0,0)$. Then $E$ can be expressed by the following equation:

$$
\mathrm{d} x^{2}+e x y+f y^{2}=1
$$

Consider the horizontal scanning line $Y=0$. This line will intersect $\mathrm{E}$ at $\mathrm{XL}_{i}(-1 / \sqrt{d}, 0)$ and $\mathrm{XR}_{i}(1 / \sqrt{d}, 0)$, and the middle point $\mathrm{XM}_{i}$ of $\mathrm{XL}_{i}$ and $\mathrm{XR}_{i}$ is $(0,0)$. Similarly, the vertical scanning line $X=0$ will intersect $\mathrm{E}$ at $\mathrm{YT}_{i}(0,1 / \sqrt{f})$ and $\mathrm{YB}_{i}(0,-1 / \sqrt{f})$, and the midpoint $\mathrm{YM}_{i}$ of $\mathrm{YT}_{i}$ and $\mathrm{YB}_{i}$ is $(0,0)$. Since $(0,0)$ is on

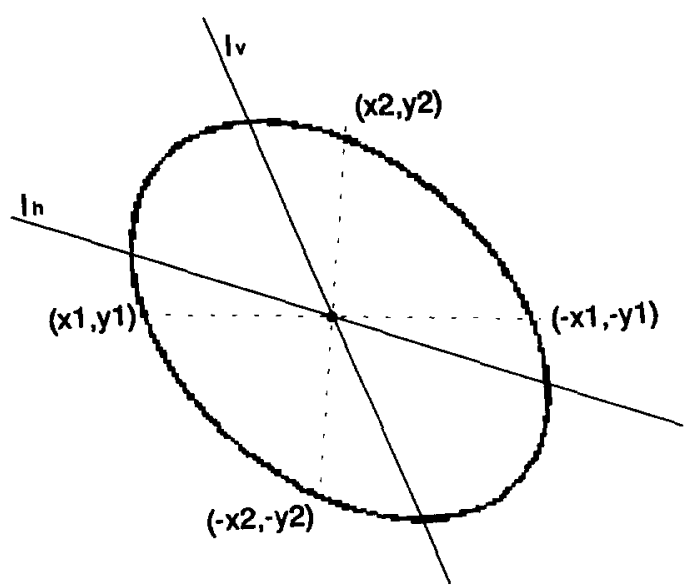

Fig. 2. The crosspoint of $l_{h}$ and $l_{v}$ is the center of the ellipse. 


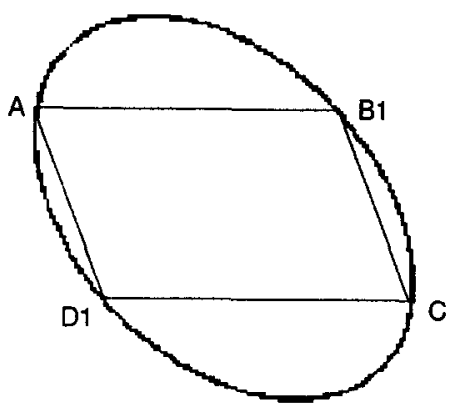

(a)

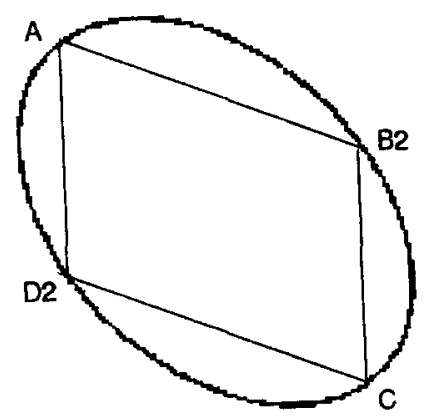

(b)

Fig. 3. Boundary point $A$ and its symmetric point $C$ relative to the center of ellipse $\mathrm{E}$ and their symmetric points relative to the symmetric vertical and horizontal axes of $E$ form two parallelograms with their vertexes on the ellipse: (a) AB1 CD1, (b) AB2CD2.

both line $l_{\mathrm{v}}$ and $l_{\mathrm{h}},(0,0)$ must be the cross-point of $l_{\mathrm{v}}$ and $l_{h}$.

Note that if the center of ellipse (or circle) $\mathrm{E}$ is $\left(x_{0}, y_{0}\right)$ and $\mathrm{E}$ is translated by $\left(-x_{0},-y_{0}\right)$, for any point $\mathrm{A}(x, y)$ on the translated $\mathrm{E}$, its symmetric point $\mathrm{C}(-x,-y)$ relative to $(0,0)$ should also be on the translated $E$ (see Fig. 2).

Theorem 4. Let $\mathrm{E}$ be an ellipse or a circle with center $(0,0), A$ be a point on $E$, and $C$ be the symmetric point of $A$ relative to $(0,0)$. Let $B_{1}$ and $D_{1}$ be the symmetric points of $\mathrm{A}$ and $\mathrm{C}$ relative to $l_{v}$, respectively. Let $\mathrm{B}_{2}$ and $D_{2}$ be the symmetric points of $C$ and $A$ relative to $l_{\mathrm{h}}$, respectively. Then the quadrangles $A \mathrm{~B}_{1} \mathrm{CD}_{1}$ and $\mathrm{AB}_{2} \mathrm{CD}_{2}$ are parallelograms (see Fig. 3).

Proof. Let the coordinates of $\mathrm{A}$ be $(u, v)$. Then $\mathrm{C}$ will be on $\mathrm{E}$ with coordinates $(-u,-v)$. Since $\mathrm{B}_{1}$ is the symmetric point of $\mathrm{A}$ relative to $l_{v}, \mathrm{~B}_{1}$ will be on the same horizontal scanning line as $A$ and have the coordinates $\left(u^{\prime}, v\right)$. Similarly, $\mathrm{D}_{1}$ will have the coordinates $\left(u^{\prime \prime},-v\right)$. Since the symmetric point $\mathrm{SB}\left(-u^{\prime},-v\right)$ of $\mathrm{B}_{1}$ relative to $(0,0)$ is on $E$ and the scanning line $Y=-v$ can intersect $E$ at only two points, $S B$ will be $D_{1}$ or $C$. Since $u^{\prime} \neq u, \mathrm{SB}$ must be $\mathrm{D}_{1}$ and $u^{\prime \prime}=-u$. Thus the lengths of line segments $\overline{\mathrm{AB}_{1}}$ and $\overline{\mathrm{CD}_{1}}$ are both $\left|u^{\prime}-u\right|$ and the quadrangle $A B_{1} C D_{1}$ is a parallelogram. In a similar way, we can prove that $\mathrm{AB}_{2} \mathrm{CD}_{2}$ is also a parallelogram.
Note that if $E$ is a circle or an ellipse without rotation, $B_{1}$ and $B_{2}$ will be the same point, $D_{1}$ and $D_{2}$ are also the same.

\subsection{Phase 1: symmetric center location}

In this subsection, based on Theorems 1-3 above, we will present the first phase of the proposed method, in which all possible centers of circles and ellipses will be located.

Let $\mathrm{f}$ be an input image. Before applying the proposed method, we first use an edge extractor ${ }^{(8)}$ to find the boundary points of objects in $f$ and store the result on an image F. Then Phase 1 is carried out. First $F$ is classified into several subimages by a horizontal scanning transform procedure, described as follows.

\subsubsection{Horizontal scanning transform procedure}

Initialize a blank image $G$

Scan F from left to right and top to bottom

For each boundary point $(i, j)$ on $\mathrm{F}$

If there exists another boundary point $(k, j)$ on $\mathrm{F}$

then for each such a point $(k, j)$, set

$$
\begin{aligned}
& \mathrm{u}=(i+k) / 2] . \\
& \mathrm{G}(u, \mathrm{j})=1
\end{aligned}
$$

End (scan)

Apply Hough transform to $G$ to extract all lines in $G$ Consider each extracted line to be a candidate symmetric vertical axis of an ellipse or a circle

For each extracted line $l_{v}$

Group all symmetric points in F relative to $l_{\mathrm{v}}$ into a subimage $F_{h}$

By Theorem 1, we know that if there exists an ellipse (or a circle) $\mathrm{E}$ in image $\mathrm{F}$, through the above procedure, each point in the left part of E must produce one point, which is on the symmetric vertical axis $l_{\mathrm{v}}$ of $\mathrm{E}$, in image G. Thus, after the above procedure, many points on $l_{v}$ should appear in G. Based on this fact, by applying Hough transform to $G$, we can extract $l_{v}$. Also, all points in $\mathrm{E}$ must be put in the same subimage. Note that ellipses with different symmetric vertical axes will be put in different subimages, which will facilitate the detection later.

Next, a vertical scanning transform is applied to each $F_{h}$ produced by the above procedure, as described below.

\subsubsection{Vertical scanning transform procedure}

Initialize a blank image $\mathrm{G}$

Scan $F_{h}$ from top to bottom and left to right

For each boundary point $(i, j)$

If there exists another boundary point $(i, k)$ in the same vertical scanning line

then for each point $(i, k)$, set

$$
\begin{aligned}
& u=[(j+k) / 2] \\
& \mathrm{G}(i, u)=1
\end{aligned}
$$

End (scan)

Apply Hough transform to $\mathrm{G}$ to extract all lines in $\mathbf{G}$ Consider each extracted line to be a candidate sym- 


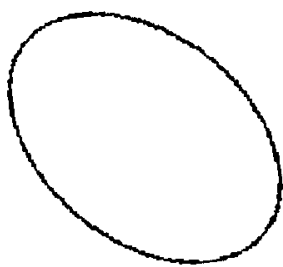

(a)

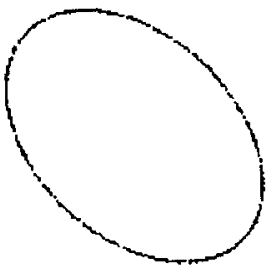

(c)

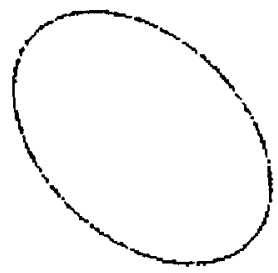

(e)

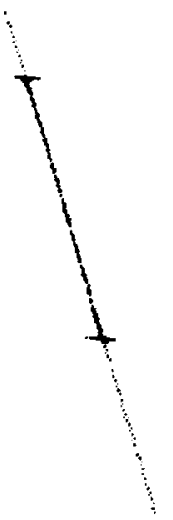

(b)

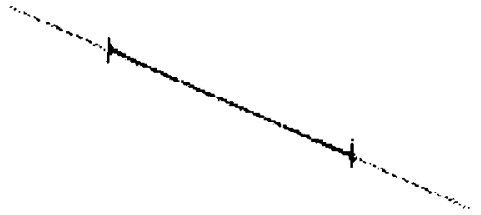

(d)

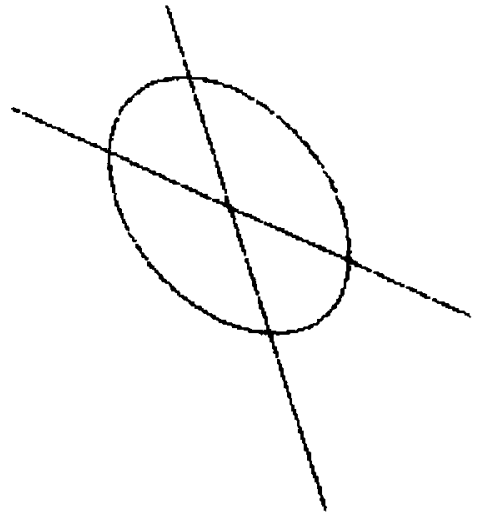

(f)

Fig. 4. An illustration of the symmetric center location phase. (a) A synthetic image. (b) A candidate symmetric vertical axis $l_{v}$ is extracted by applying the horizontal scanning transform to (a). (c) The corresponding symmetric boundary points relative to $l_{v}$. (d) A candidate symmetric horizontal axis $l_{h}$ is extracted by applying the vertical scanning transform to (c). (e) The corresponding symmetric boundary points relative to $l_{h}$. (f) The crosspoint of $l_{h}$ and $l_{v}$ is the center of the ellipse.

metric horizontal axis of an ellipse or a circle

For each extracted line $l_{\mathrm{h}}$

Group all symmetric points in $F_{h}$ relative to $l_{h}$ into a subimage $F_{h v}$

Consider the cross point of $l_{\mathrm{h}}$ and $l_{\mathrm{v}}$ to be a candidate center of an ellipse or a circle.

Note that if there exists an ellipse $E$ in $F$ with symmetric axes $l_{\mathrm{h}}$ and $l_{\mathrm{v}}$, then through the above two procedures, $l_{\mathrm{h}}$ and $l_{\mathrm{v}}$ will be extracted and all points in $E$ will be put in the same subimage. Furthermore, by Theorem 3, we know that the cross-point of $l_{\mathrm{h}}$ and $l_{\mathrm{v}}$ must be the center of $E$. Based on this fact, each cross-point of the lines $l_{\mathrm{h}}$ and $l_{\mathrm{v}}$ can be considered to be a candidate center of an ellipse or a circle.

In Fig. 4 an example is given to illustrate the symmetric center location phase. Applying the horizontal scanning transform procedure to Fig. 4(a), one candi- date symmetric vertical axis $l_{v}$ is extracted and shown in Fig. 4(b), the corresponding symmetric boundary points with respect to $l_{v}$ are shown in Fig. $4($ c). Figure $4(d)$ shows a candidate symmetric horizontal axis $l_{\mathrm{h}}$ extracted by applying the vertical scanning transform procedure to Fig. 4(c). Figure 4(e) shows the corresponding symmetric boundary points with respect to $l_{\mathrm{h}}$. The crosspoint of $l_{\mathrm{h}}$ and $l_{\mathrm{v}}$ is considered to be a candidate for the center of the ellipse, as shown in Fig. 4(f).

\subsection{Phase 2: parameter estimation}

In Phase 2, based on the result of Phase 1 and Theorems 3-4, the remaining three parameters ( $a$ : halflength of the major axis, $b$ : half-length of the minor axis, $\theta$ : the orientation angle) for each ellipse or the single parameter (radius) for a circle in each subimage $F_{h v}$ will be found. Initially, the proposed method es- 
tablishes an array $\operatorname{AR}(a, b, \theta)$ with $\operatorname{AR}(a, b, \theta)=0$ for each entry $(a, b, \theta)$. Then, for each subimage $\mathrm{F}_{\mathrm{hv}}$ corresponding to a candidate symmetric center $O\left(x_{0}, y_{0}\right)$, each point $A$ in $F_{h v}$ is checked to see if its symmetric point $C$ relative to $O$ exists in $F_{h y}$. If it does, then $B_{1}$ and $D_{1}$, the points in $F_{h v}$ that are symmetric to $A$ and $\mathrm{C}$, respectively, with respect to $l_{v}$, are located. $\mathrm{B}_{2}$ and $D_{2}$, the points symmetric to $C$ and $A$ with respect to $l_{\mathrm{h}}$, are located. Then the two quadrangles $\mathrm{AB}_{1} \mathrm{CD}_{1}$ and $\mathrm{AB}_{2} \mathrm{CD}_{2}$ are checked to see whether they are parallelograms (by testing if the length of $\overline{\mathbf{A B}_{1}}$ equals that of $\overline{\mathrm{CD}_{1}}$ and if the length of $\overline{\mathrm{AD}_{2}}$ equals that of $\overline{\mathrm{B}_{2} \mathrm{C}}$ ). If they are, points $A, C, B_{1}, B_{2}, D_{1}$, and $D_{2}$ are considered to lie on an ellipse. Then $A, B_{1}$, and $B_{2}$ are shifted $\left(-x_{0},-y_{0}\right)$ to move the ellipse center to $(0,0)$.

Since an ellipse with center $(0,0)$ can be expressed by equation (1), by substituting the coordinates of the shifted $A, B_{1}$, and $B_{2}$ into equation (1), we can obtain three equations with three unknown parameters $(d, e, f)$. Thus once these three parameters $(d, e, f)$ are solved, the three parameters $(a, b, \theta)$ for the ellipse can be obtained by the following formulas: ${ }^{(9)}$

$\theta=\left\{\tan ^{-1}[e /(d-f)]\right\} / 2$

$a=\operatorname{sqrt}\left[1 /\left(d \times \cos ^{2} \theta+e \times \sin \theta \times \cos \theta+f \times \sin ^{2} \theta\right)\right]$

$b=\operatorname{sqrt}\left[1 /\left(f \times \cos ^{2} \theta-e \times \sin \theta \times \cos \theta+d \times \sin ^{2} \theta\right)\right]$.

The $(a, b, \theta)$ obtained is then considered to be a set of possible parameters for an ellipse and the corresponding entry $\operatorname{AR}(a, b, \theta)$ is increased by one. After all points in $F_{\mathrm{hv}}$ are processed, a local peak-finding algorithm is finally applied to the array $\operatorname{AR}(a, b, \theta)$ and the peaks that are found are considered to be the parameters of ellipses. Note that if there exists an ellipse $E$ in $F_{h v}$ with parameters $\left(a_{0}, b_{0}, \theta_{0}, x_{0}, y_{0}\right)$ in $\mathrm{F}_{\mathrm{hv}}$, each point $\mathrm{A}$ on $\mathrm{E}$ should contribute one to $\operatorname{AR}\left(a_{0}, b_{0}, \theta_{0}\right)$. Hence, after the above processes, the value of $\operatorname{AR}\left(a_{0}, b_{0}, \theta_{0}\right)$ must be large and the ellipse can be found through the local
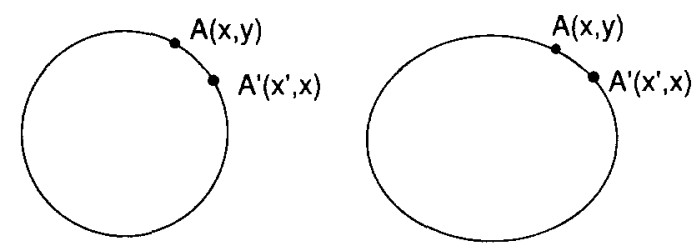

(a)
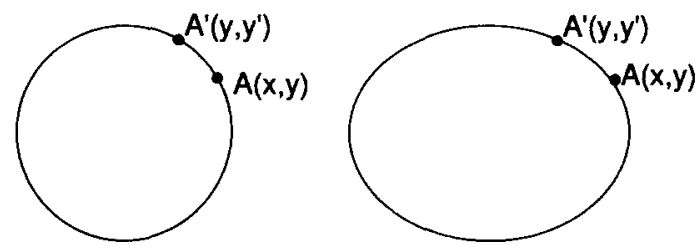

(b)

Fig. 5. Using $\mathrm{A}(x, y)$ and $\mathrm{A}^{\prime}$ to estimate a circle or no-orientation ellipse: (a) $\mathrm{A}^{\prime}=\left(x^{\prime}, x\right)(|x|<|y|)$; (b) $\mathrm{A}^{\prime}=\left(y, y^{\prime}\right)(|x| \geq|y|)$. peak-finding algorithm. The details of Phase 2 are described as follows:

Initialize a blank array $\operatorname{AR}(a, b, \theta)$

For a point $A$ in $F_{h v}$

If its symmetric point $C$ with respect to $\mathrm{O}\left(x_{0}, y_{0}\right)$ is not in $F_{h v}$, give up the point $A$

else

By $A$ and $C$ form two quadrangles $A B_{1} C D_{1}$ and $\mathrm{AB}_{2} \mathrm{CD}_{2}$

If $A B_{1} C D_{1}$ and $A B_{2} C D_{2}$ are not parallelograms, give up $A$ else

Use $A, B_{1}$ and $\mathbf{B}_{2}$ to solve equation (1), obtain $(d, e, f)$

Use equation (2) to obtain $(a, b, \theta)$

$\operatorname{AR}(a, b, \theta)=\mathbf{A R}(a, b, \theta)+1$

The peak-finding algorithm is applied to AR, and the peaks found are considered to be the parameters of ellipses.

Note that if the detected shape is a circle (i.e. $a=b$, $\theta=0$ ) or an ellipse with $\theta=0$, we will find that $B_{1}=B_{2}$, as mentioned previously, and the above procedure cannot be used to solve for the parameters. In this situation, the shifted circle or ellipse with center $(0,0)$ can be expressed by

$$
\mathrm{d} x^{2}+f y^{2}=1 .
$$

To solve $(d, f)$, for point $\mathrm{A}(x, y)$, take each point $\mathrm{A}^{\prime}$ (see Fig. 5) in $F_{h v}$ with

$$
\begin{array}{ll}
\mathrm{A}^{\prime}=\left(x^{\prime}, x\right) & \text { if }|x|<|y| \\
\mathrm{A}^{\prime}=\left(y, y^{\prime}\right) & \text { otherwise. }
\end{array}
$$

By substituting the coordinates of the shifted $\mathrm{A}$ and $\mathrm{A}^{\prime}$ into equation (3), we can solve $(d, f)$. After we have $(d, f)$, the parameters $(a, b)$ can be obtained by

$$
\begin{aligned}
& a=\operatorname{sqrt}(1 / d) \\
& b=\operatorname{sqrt}(1 / f) .
\end{aligned}
$$

The obtained parameters $(a, b)$ are then considered to be a set of possible parameters for an ellipse without any orientation or a circle. The remaining steps are similar to the procedure used for an ellipse with orientation except that array $\operatorname{AR}(a, b, \theta)$ is replaced by $\operatorname{AR} 1(a, b)$.

\section{EXPERIMENTAL RESULTS}

This section presented the results of experiments in which the proposed method was applied to several images. Figure 6(a) shows a synthetic image including four objects: two overlapping ellipses, a circle, and an ellipse without orientation. In the symmetric center location phase, four symmetric centers were extracted and their corresponding symmetric boundary points were put into four different subimages, as shown in Fig. 6(b)-(e). The parameter estimation phase was then applied to each subimage to find the remaining parameters. Figure 6(f) presents the final result super- 


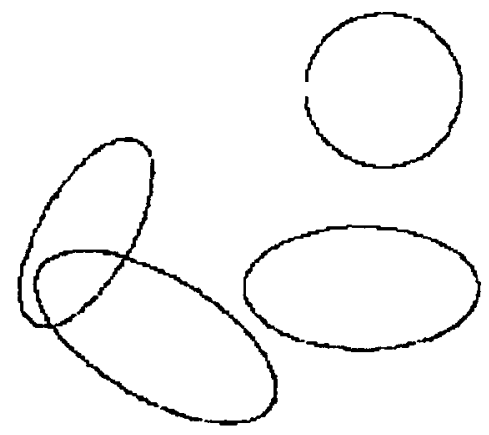

(a)

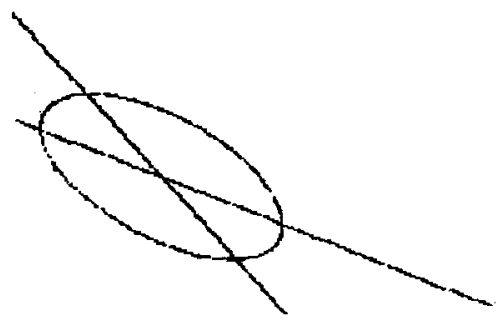

(c)

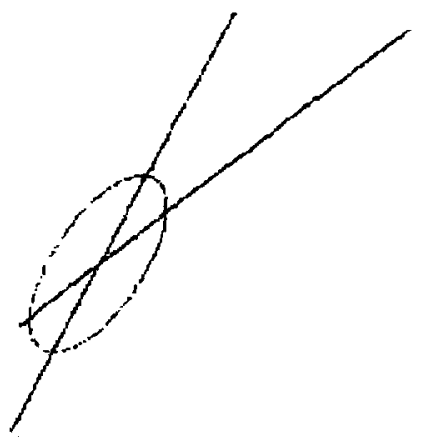

(b)

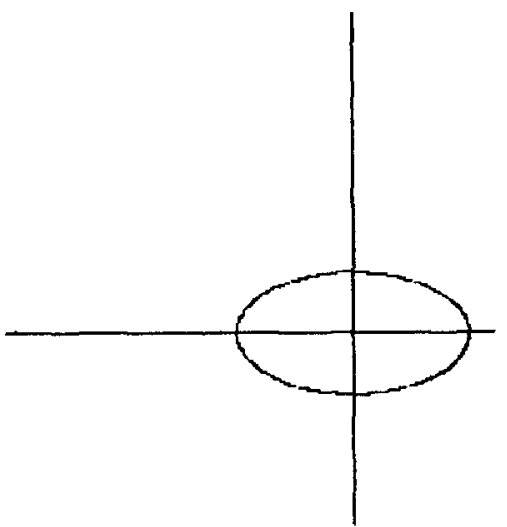

(d)

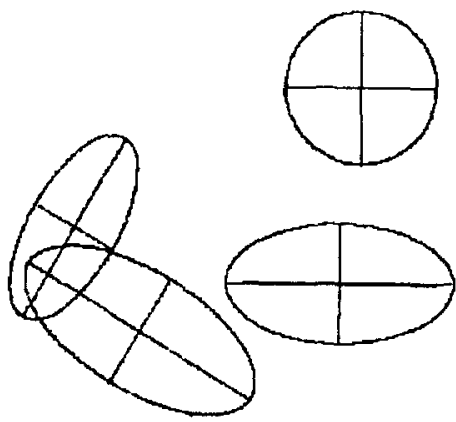

(f)

Fig. 6. Results of applying the proposed method to a synthetic image. (a) A synthetic image. (b)-(e) The results of applying symmetric center location phase to (a): four subimages with their corresponding symmetric centers are obtained. (f) The final result of the proposed method.

imposed on the original image with a set of two orthogonal-cross line segments standing for the major and minor axes of the ellipses. Figure $7($ a) shows a real image including a circle, an ellipse, and some other types of objects. Figure 7(b) shows the result of boundary extraction. Applying the proposed method to Fig. 7(b) yielded two subimages corresponding to the two centers, as shown in Fig. 7(c)-(d). The final result is shown in Fig. 7(e). From these experimental results, we can see that separated and partially occluded ellipses (circles) can be located successfully. For comparison purposes, we also apply the Tsuji method ${ }^{(2)}$ to Fig. 7(a). By using a SUN SPARC-10 workstation, the running time for our method is $7.9 \mathrm{~s}$, and for the Tsuji method is $8.9 \mathrm{~s}$. The proposed method is little faster than the Tsuji method. But the Tsuji method needs the edge direction information, it is a hard work to get accurate edge direction in a noisy image.

Figure 8(a) shows another synthetic image including four ellipses: (1) intact, (2) intermittent, (3) with $12.5 \%$ 


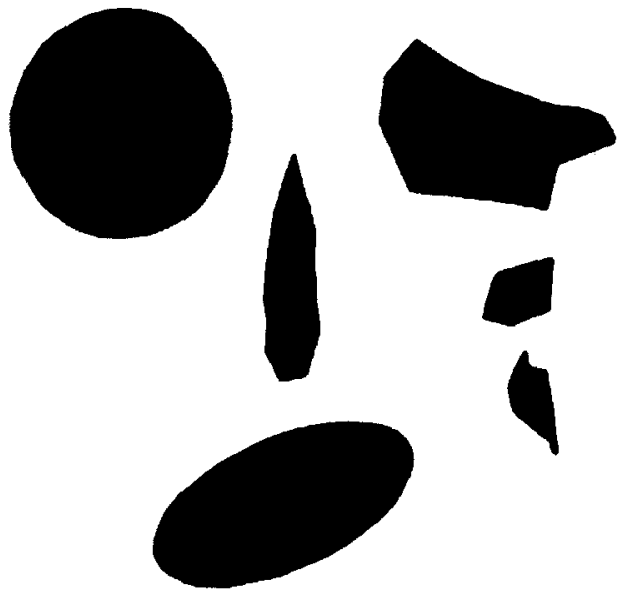

(a)

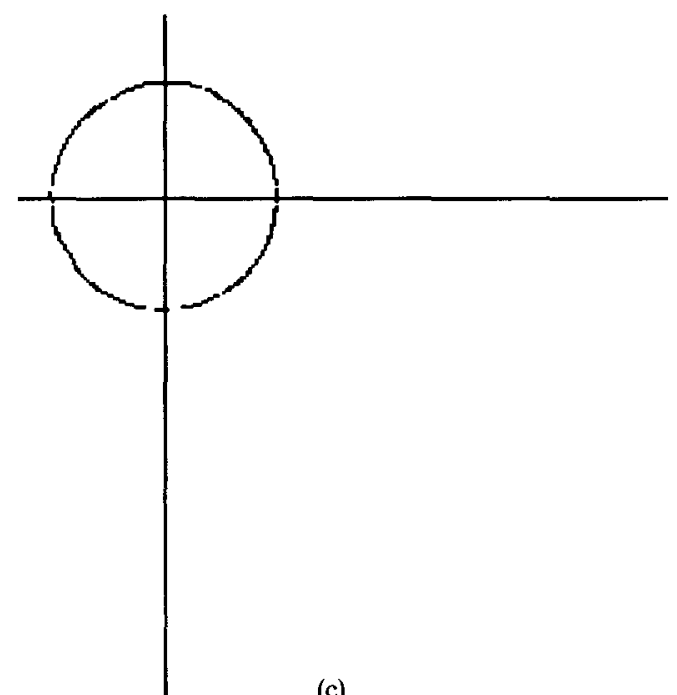

(c)

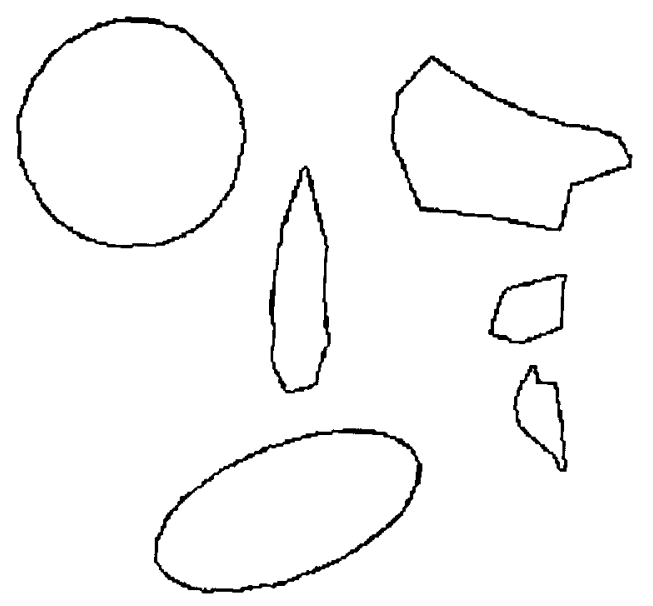

(b)

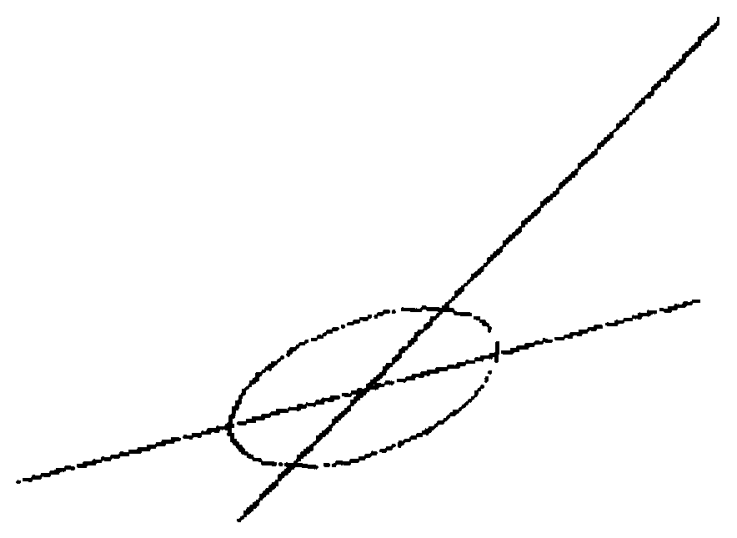

(d)

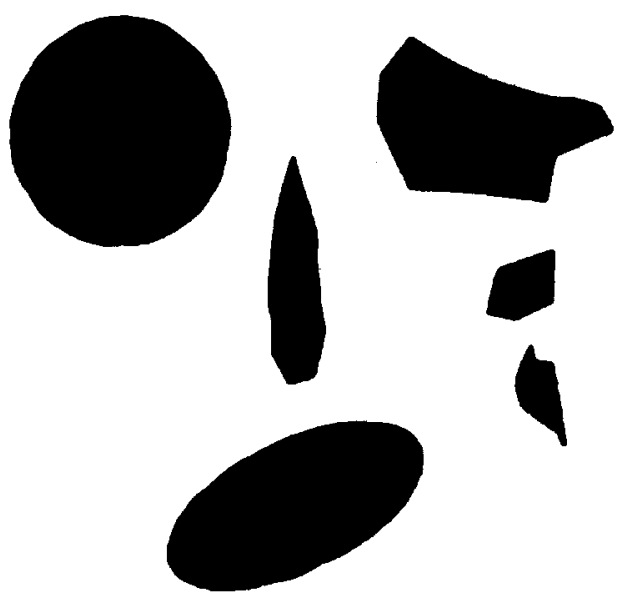

(e)

Fig. 7. Results of applying the proposed method to a real image. (a) A real image. (b) The result of boundary extraction. (c) and (d) The results of applying symmetric center location phase to (b): two subimages with their corresponding symmetric centers are obtained. (e) The final result of the proposed method. 

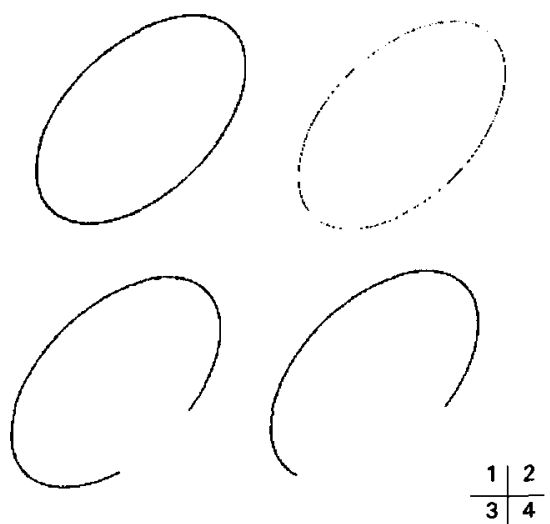

(a)
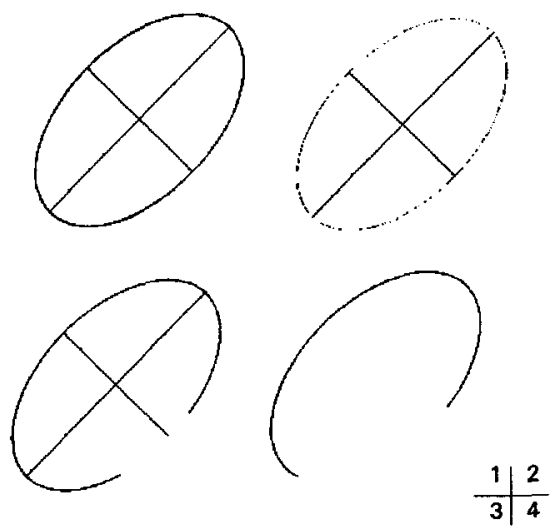

(b)

Fig. 8. Results of applying the proposed method to defective ellipses. (a) Four ellipses: intact, intermittent, $12.5 \%$ defect and $25 \%$ defect. (b) Three ellipses are detected, the ellipse with $25 \%$ defect is not located.

defect, (4) with $25 \%$ defect. The detecting results are shown in Fig. 8(b). From this figure, we can see that ellipse with $25 \%$ defect is not detected. This is due to that many points on the boundary of the ellipse cannot be used to form parallelograms of the type employed in Phase 2. In general, if the defectiveness of an ellipse exceeds $25 \%$, the proposed method may not work well.

\section{CONCLUSION}

In this paper, we have proposed a fast method for locating circles and ellipses. The symmetry of ellipses and circles is used as a basis for classifying boundary points into several subimages, each of which is then treated separately. In Phase 2, because we again take advantage of global geometric symmetry, for each boundary point $A$, we only need to evaluate one set of parameters $(a, b, \theta)$ of an ellipse that possibly passes through A. Since the edge direction is not used, the estimation accuracy will not be lost. Thus, the proposed method is faster and more precise than existing Houghbased algorithms for ellipse and circle detection. In addition, the proposed method is simple and easy to implement.

Acknowledgment - We would like to acknowledge the generous support by the National Science Council of R.O.C. under contract NSC-82-0408-E-009-425. In addition, we would also like to express our appreciation to the referees for their many valuable suggestions that greatly improved the readability and interest of this paper.

\section{REFERENCES}

1. P. V. C. Hough, Method and means for recognizing complex pattern, U.S. Patent No. 3069654 (1962).

2. S. Tsuji and M. Matsumoto, Detection of ellipses by a modified Hough transform, IEEE Trans. Comput. 27, 777-781 (1978).

3. D. H. Ballard, Generalizing the Hough transform to detect arbitrary shapes, Pattern Recognition, 13, 111-122(1981).

4. H. K. Yune, J. Illingworth and J. Kittler, Detection partially occluded ellipses using the Hough transform, Image and Vision Computing 7, 31-37 (1989).

5. J. Illingworth and J. Kittler, The adaptive Hough transform, IEEE Trans. Patt. Anal. Mach. Intell. 10, 690-698 (1987).

6. K. K. Yip Raymond, K. S. Tam Peter and N. K. Leung Dennis, Modification of Hough transform for circles and ellipses detection using a 2-dimensional array, Pattern Recognition, 25, 1007-1022 (1992).

7. P. Y. Yin and L. H. Chen, A new method for ellipses detection using symmetry, J. Electronic Imaging 3, 20-29 (1994).

8. R. C. Gonzalez and P. Wintz, Digital Image Processing. Addision Wesley, Reading, Massachusetts (1987).

9. W. Wernick, Analytic Geometry, Silver Burdett Company (1968).

\footnotetext{
About the Author-CHUN-TA HO received the B.S. degree in Computer and Information science from National Chiao Tung University, Taiwan, in 1991. Since then, he has been a Ph.D. student at the Institute of Computer and Information science at National Chiao Tung University. His current research interests include image processing and pattern recognition.
}

\begin{abstract}
About the Author-LING-HWEI CHEN received the B.S. degree in mathematics and the M.S. degree in applied mathematics from National Tsing Hua University, Taiwan, in 1975 and 1977, respectively, and Ph.D. degree in computer engineering from National Chiao-Tung University, Taiwan, in 1987. From 1977 to 1979 , she worked as a research assistant in Chung-Shan Institute of Science and Technology, Taiwan. From 1979 to 1981, she worked as a research associate at Electronic Research and Service Organization, Industry Technology Research Institute, Taiwan. From 1981 to 1983 , she worked as an engineer at the Institute of Information Industry, Taiwan. She joined the Department of Computer and Information Science at National Chiao Tung University in 1987 and is currently a professor there. Her current research interests include image processing and pattern recognition.
\end{abstract}

